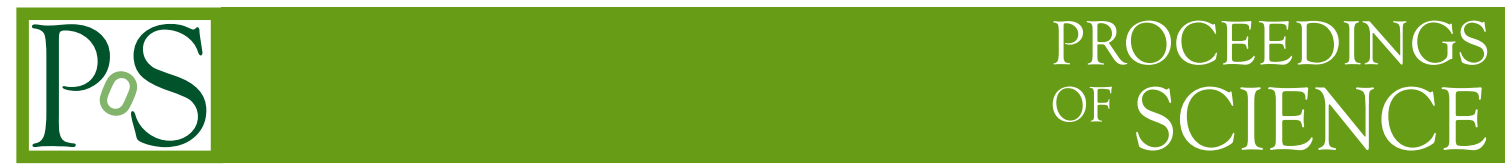

\title{
Where we are on B-physics discrepancies
}

\author{
Diego Guadagnoli* \\ LAPTh, CNRS \& Université de Savoie Mont-Blanc, 74941 Annecy, France \\ E-mail: diego.guadagnoli@lapth.cnrs.fr
}

\begin{abstract}
We discuss the theory interpretation of the discrepancies in semi-leptonic $B$ decays as of Moriond 2019. By critically including loop-induced effects that have not been discussed before in the context of global fits, we show that a fully coherent picture is possible all the way from the weakeffective-theory to the SMEFT to the simplified-model level.
\end{abstract}

Corfu Summer Institute 2019 "School and Workshops on Elementary Particle Physics and Gravity" (CORFU2019)

31 August - 25 September 2019

Corfù, Greece

${ }^{*}$ Speaker. 


\section{Introduction}

Recent years witnessed the build-up of several deviations from Standard-Model (SM) expectations in semi-leptonic $B$ decays. These deviations fall in four groups of datasets, each of which characterized by different measurements, and different theory and experimental challenges:

(a) $b \rightarrow s \mu \mu$ differential branching-ratio data lower than the corresponding SM predictions [1, 2]. Here the main challenge is the control over $B$ to light meson hadronic form factors $[3,4,5]$;

(b) Deviations with respect to SM predictions in $B \rightarrow K^{*} \mu^{+} \mu^{-}$angular observables in certain kinematic regions for the di-lepton $q^{2}[6,7,8,9]$. Although form factor uncertainties are under better control than for branching ratios, hadronic uncertainties are nevertheless significant $[10,11]$ (see however [12]);

(c) Deviations from lepton universality in $b \rightarrow s \ell \ell$ transitions in the processes $B \rightarrow K \ell \ell$ and $B \rightarrow$ $K^{*} \ell \ell$ (via the $\mu / e$ ratios $R_{K}$ [13] and $R_{K^{*}}$ [14]). Here the challenge is mostly statistics, due in particular to the $e e$ channel, that requires harder $p_{T}$ thresholds and has larger bremsstrahlung systematics;

(d) Deviations from $\tau-\mu$ and $\tau-e$ universality in $b \rightarrow c \ell v$ transitions $[15,16,17,18,19,20$, 21]. Here uncertainties, besides statistics, include a non-negligible experimental systematics, whereas theory uncertainties are small [22, 23, 24, 25].

It is quite impressive that items $(a)$ to $(c)$ can be simultaneously explained with one and the same shift to two four-fermion semi-leptonic operators and as a result, the picture be substantially improved with respect to the SM. It is likewise quite enticing that all items $(a)$ to $(d)$ can be explained, not only qualitatively but even quantitatively, all the way from the level of the Weak Effective Theory (WET), to the so-called SM Effective Field Theory (SMEFT), to the level of simplified models, as shown in Ref. [26].

Ref. [26]'s conclusions rely on a number of data updates, among the others:

(1) The new measurement of $R_{K}$ by the LHCb collaboration combining Run-1 data with $2 \mathrm{fb}^{-1}$ of Run-2 data (corresponding to about one third of the full Run-2 data set) [27].

The SM predicts lepton flavour universality, i.e. $R_{K}^{\mathrm{SM}}$ is unity with uncertainties [28] that are well below the current experimental sensitivities. While the updated experimental value is closer to the SM prediction than the Run-1 result [13], the reduced experimental uncertainties imply a tension between theory and experiment at the level of $2.5 \sigma$, which is comparable to the situation before the update.

(2) The new, preliminary measurement of $R_{K^{*}}$ by Belle [29].

Averaged over $B^{ \pm}$and $B^{0}$ decays, the measured $R_{K^{*}}$ values at low and high $q^{2}$ are

$$
R_{K^{*}}=\frac{\mathrm{BR}\left(B \rightarrow K^{*} \mu \mu\right)}{\mathrm{BR}\left(B \rightarrow K^{*} e e\right)}= \begin{cases}0.90_{-0.21}^{+0.27} \pm 0.10, & \text { for } 0.1 \mathrm{GeV}^{2}<q^{2}<8 \mathrm{GeV}^{2} \\ 1.18_{-0.32}^{+0.52} \pm 0.10, & \text { for } 15 \mathrm{GeV}^{2}<q^{2}<19 \mathrm{GeV}^{2}\end{cases}
$$


Given their sizable uncertainties, these values are compatible with both the SM predictions and previous results on $R_{K^{*}}$ from $\mathrm{LHCb}$ [14]

$$
R_{K^{*}}=\frac{\mathrm{BR}\left(B \rightarrow K^{*} \mu \mu\right)}{\mathrm{BR}\left(B \rightarrow K^{*} e e\right)}= \begin{cases}0.66_{-0.07}^{+0.11} \pm 0.03, & \text { for } 0.045 \mathrm{GeV}^{2}<q^{2}<1.1 \mathrm{GeV}^{2} \\ 0.69_{-0.07}^{+0.11} \pm 0.05, & \text { for } 1.1 \mathrm{GeV}^{2}<q^{2}<6 \mathrm{GeV}^{2}\end{cases}
$$

that are in tension with the SM predictions by $\sim 2.5 \sigma$ in both $q^{2}$ bins.

(3) One further, important piece of information included in our study is the 2018 measurement of $B_{s} \rightarrow \mu \mu$ by the ATLAS collaboration [30], that we combine with the existing measurements by CMS and LHCb $[31,32,33]$. (Later experimental updates are not included as of this writing.)

We refer the reader to Ref. [26] for full details.

\section{Weak Effective Theory}

The first step is to address the question whether these datasets can be described within the most general effective theory constructed at the electroweak scale. As well known, this is not only possible, but even rather simple to accomplish. Data obey a pattern, which quite clearly suggests that new effects may involve two dominant structures: a left-handed $\bar{b} s$ current times a vector $\left(\mathscr{O}_{9}\right)$ or an axial-vector muon current $\left(\mathscr{O}_{10}\right)$. The best performing new-physics scenarios to explain the data involve precisely these two operators, and in particular either $\mathscr{O}_{9}$ alone, or the combination $\mathscr{O}_{9}-\mathscr{O}_{10}$, yielding a $(V-A) \times(V-A)$ structure, well suited to UV interpretations.
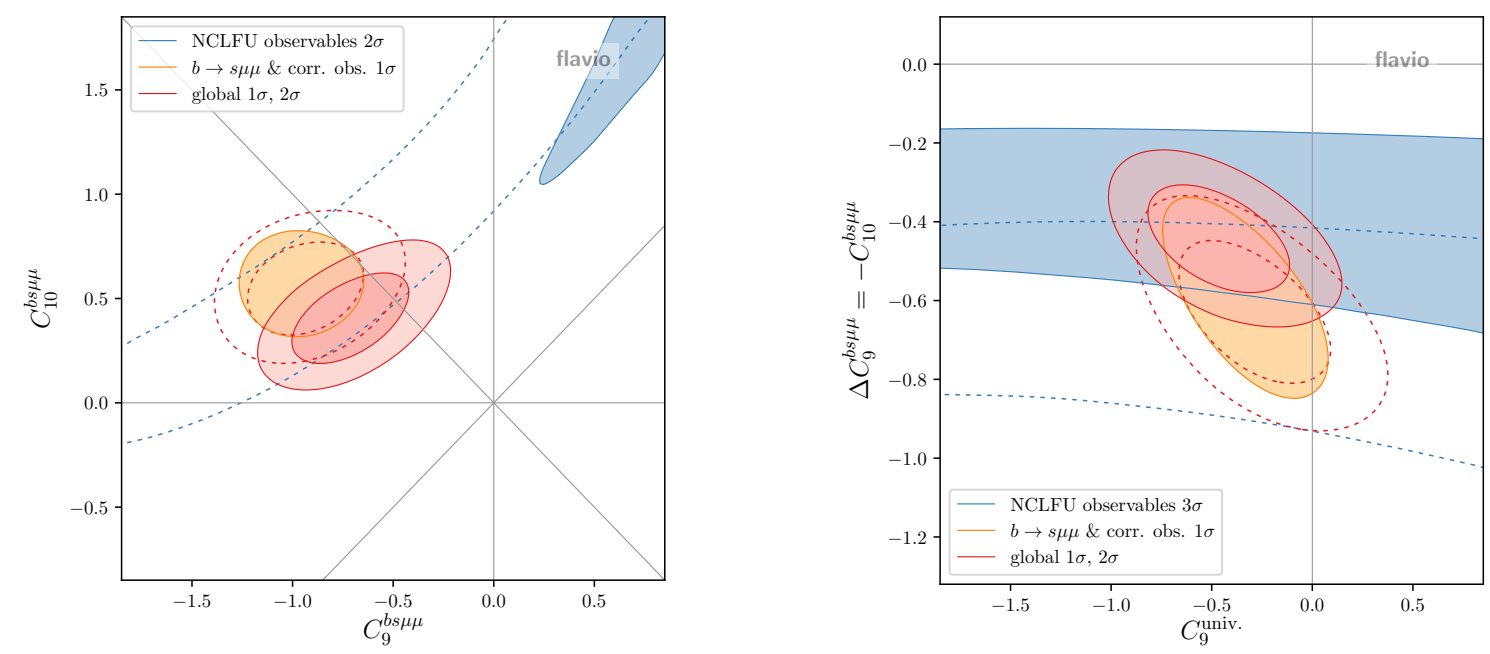

Figure 1: Neutral-current lepton flavour universality (NCLFU) observables (blue), $b \rightarrow s \mu \mu$ and correlated observables (yellow) and global fit (red). Dashed contours exclude the Moriond-2019 results.

At the quantitative level, one may first try with a single d.o.f. left floating, and fit it to data. In this case, two scenarios stand out, either $C_{9}^{(\mu)}$ alone or $C_{9}^{(\mu)}=-C_{10}^{(\mu)}$. This was the case also 
before Moriond 2019. However, at least within the approach of [26], the second scenario has an appreciably better performance with respect to the former. This is due to a concurrence of causes, notably the fact that $\mathscr{B}\left(B_{s} \rightarrow \mu \mu\right)$ prefers more and more a non-zero shift to $C_{10}^{(\mu)}$, as shown in the $C_{9}^{(\mu)}$ vs. $C_{10}^{(\mu)}$ plane in Fig. 1 . We see that $R_{K^{(*)}}$ and $b \rightarrow s \mu \mu$ data perfectly overlapped before the Moriond-2019 update. Thereafter, these two regions are in much lesser agreement, especially in the $C_{9}$ direction. There is likewise a slight tension between $R_{K}$ and $R_{K^{*}}$, which may be addressed with right-handed quark currents. Thus one would be tempted to advocate e.g. $O_{9}^{(\mu) \prime}$. However, such operator would not accommodate $B_{s} \rightarrow \mu \mu$.

One important point is that, as mentioned, in the $C_{9}^{(\mu)}$ vs. $C_{10}^{(\mu)}$ plane there is a degree of tension between $R_{K^{(*)}}$ and $b \rightarrow s \mu \mu$ data, and a lepton-universal shift to $C_{9}$ would shift $b \rightarrow s \mu \mu$ but not ratio data.

It is thus interesting to consider the case of $\Delta C_{9}^{(\mu)}=-C_{10}^{(\mu)}$ vs. $C_{9}^{\text {univ. }}$, displayed in the right panel of Fig. 1. Before Moriond 2019, the $R_{K}$ band was lower, and overlapped with $b \rightarrow s \mu \mu$ in a region with zero $C_{9}^{\text {univ. }}$. After Moriond, accord between the two datasets prefers a non-zero $C_{9}^{\text {univ. }}$. As we will see, such occurrence admits a well-defined UV interpretation.

\section{SMEFT}

We next discuss the performance of an EFT description at a scale of a few $\mathrm{TeV}$, and without new d.o.f. beyond those in the SM. Effects beneath such new scale are described by the SMEFT. In our case, contributions to the two directions identified before, namely $\Delta C_{9}^{(\mu)}=-C_{10}^{(\mu)}$ and $C_{9}^{\text {univ. }}$, can come from: (i) these very semi-leptonic operators, constructed out of left-handed SM multiplets, $L$ and $Q$, and either singlets or triplets under $S U(2)_{L}$, i.e. $\bar{L}^{(3)} \gamma^{\alpha} L^{(3)} \bar{Q}^{(2)} \gamma_{\alpha} Q^{(3)}$

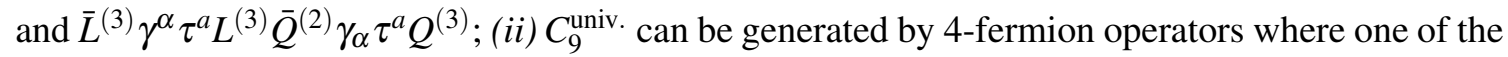
two bilinears is closed in a loop to which a virtual gauge boson emitting a lepton pair is attached $[34,35]$. Hence the amplitude is lepton-universal by gauge universality.

Interestingly, if the closed loop involves two $\tau$ 's, the corresponding 4-fermion operator can

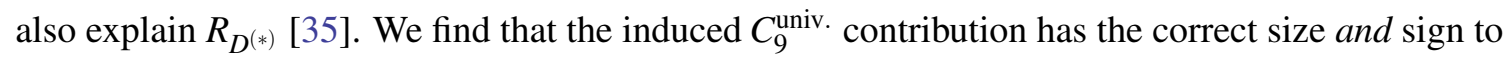
quantitatively accommodate $b \rightarrow s \mu \mu$. Then the very same operators, but with muonic indices, can explain $R_{K^{(*)}}$. The only caveat is that singlet and triplet couplings in the semi-tauonic case must be approximately equal in size in order to avoid $B \rightarrow K^{(*)} v \bar{v}$ constraints [36].

This scenario can be visualized in the first panel of fig. 2, in the plane of tauonic vs. muonic singlet-equal-to-triplet couplings. Again, before Moriond 2019, $R_{K}$ (blue) and $b \rightarrow s \mu \mu$ (yellow) were in perfect agreement in a region that however did not overlap with the $R_{D^{(*)}}$ region (green). After Moriond 2019, the blue, yellow and green regions all overlap.

\section{Simplified models}

The previous EFT picture finds a UV interpretation within the $U_{1}$ vector-leptoquark model, with $U_{1} \sim(\mathbf{3}, \mathbf{1})_{2 / 3}$ under the SM gauge group [37, 38, 39, 40, 41, 42, 43, 44, 45, 46]. This is the only single mediator that can yield non-zero values for $\left[C_{l q}^{(1)}\right]_{3323}=\left[C_{l q}^{(3)}\right]_{3323}$ and $\left[C_{l q}^{(1)}\right]_{2223}=$ $\left[C_{l q}^{(3)}\right]_{2223}$. 

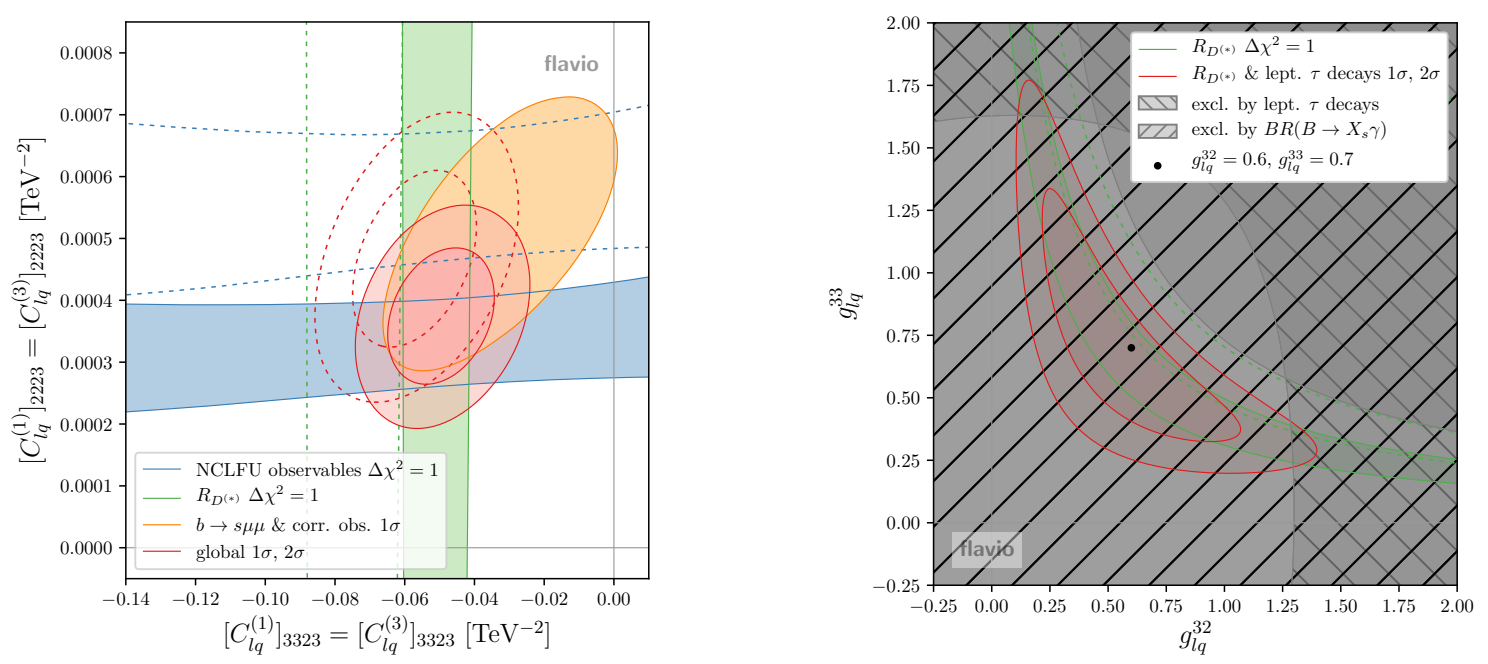

Figure 2: First panel: Neutral-current lepton flavour universality (NCLFU) observables (blue), $b \rightarrow s \mu \mu$ and correlated observables (yellow), $R_{D^{(*)}}$ (green) and global fit (red). Dashed contours exclude the Moriond2019 results. Second panel: plane of tauonic couplings within the $U_{1}$ simplified model.

One can then define the simplified-model coupling $\mathscr{L}_{U_{1}} \supset g_{l q}^{j i}\left(\bar{q}^{i} \gamma^{\mu} l^{j}\right) U_{\mu}+$ h.c.. A shift to $R_{K^{(*)}}$ will depend on $g_{l q}^{22,23}$, whereas a shift to $R_{D^{(*)}}$ depends on $g_{l q}^{33,32}$. Tauonic couplings are known to be constrained by $\tau \rightarrow \ell v v$, hence it is far from obvious that the whole picture can work quantitatively. The plane of tauonic couplings is displayed in Fig. 2 (second panel) and it shows that $R_{D^{(*)}}$ can indeed be comfortably accommodated in compliance with all constraints. We note that radiative decays provide a further constraint, which is however very model-dependent, because additional fermions would also contribute to dipole structures and shift $B \rightarrow X_{s} \gamma$ along the diagonal [47]. Choosing a benchmark point in the tauonic-couplings plane, e.g. $\left(g_{l q}^{32}, g_{l q}^{33}\right)=(0.6,0.7)$ also displayed in the figure, one can see that all constraints are fulfilled in the plane $g_{l q}^{22,23}$ of the muonic couplings [26]. In particular, the above benchmark point performs way better than the case of null tauonic couplings. Finally, this model also allows to address the question whether such tauonic couplings may be constrained by direct searches, discussed in [48, 49, 50, 51, 52, 53]. We find that the indirect constraint from leptonic $\tau$ decays $[54,55]$ is stronger than the direct constraints in nearly all of the parameter space.

\section{Acknowledgments}

I warmly thank Gui Rebelo and George Zoupanos for the kind invitation to this beautiful venue.

\section{References}

[1] LHCв collaboration, R. Aaij et al., Differential branching fractions and isospin asymmetries of $B \rightarrow K^{(*)} \mu^{+} \mu^{-}$decays, JHEP 06 (2014) 133, [1403.8044].

[2] LHCв collaboration, R. Aaij et al., Angular analysis and differential branching fraction of the decay $B_{s}^{0} \rightarrow \phi \mu^{+} \mu^{-}$, JHEP 09 (2015) 179, [1506.08777]. 
[3] A. Bharucha, D. M. Straub and R. Zwicky, $B \rightarrow V \ell^{+} \ell^{-}$in the Standard Model from light-cone sum rules, JHEP 08 (2016) 098, [1503.05534].

[4] R. R. Horgan, Z. Liu, S. Meinel and M. Wingate, Rare B decays using lattice QCD form factors, PoS LATTICE2014 (2015) 372, [1501.00367].

[5] N. Gubernari, A. Kokulu and D. van Dyk, $B \rightarrow P$ and $B \rightarrow V$ Form Factors from B-Meson Light-Cone Sum Rules beyond Leading Twist, JHEP 01 (2019) 150, [1811. 00983 ].

[6] LHCB collaboration, R. Aaij et al., Angular analysis of the $B^{0} \rightarrow K^{* 0} \mu^{+} \mu^{-}$decay using $3 f^{-1}$ of integrated luminosity, JHEP 02 (2016) 104, [1512.04442].

[7] ATLAS collaboration, Angular analysis of $B_{d}^{0} \rightarrow K^{*} \mu^{+} \mu^{-}$decays in pp collisions at $\sqrt{s}=8 \mathrm{TeV}$ with the ATLAS detector, Tech. Rep. ATLAS-CONF-2017-023, CERN, Geneva, Apr, 2017.

[8] CMS collaboration, Measurement of the $P_{1}$ and $P_{5}^{\prime}$ angular parameters of the decay $\mathrm{B}^{0} \rightarrow \mathrm{K}^{* 0} \mu^{+} \mu^{-}$ in proton-proton collisions at $\sqrt{s}=8 \mathrm{TeV}$, Tech. Rep. CMS-PAS-BPH-15-008, CERN, Geneva, 2017.

[9] CMS collaboration, V. Khachatryan et al., Angular analysis of the decay $B^{0} \rightarrow K^{* 0} \mu^{+} \mu^{-}$from $p p$ collisions at $\sqrt{s}=8 \mathrm{TeV}$, Phys. Lett. B753 (2016) 424-448, [1507.08126].

[10] A. Khodjamirian, T. Mannel, A. A. Pivovarov and Y. M. Wang, Charm-loop effect in $B \rightarrow K^{(*)} \ell^{+} \ell^{-}$ and $B \rightarrow K^{*} \gamma$, JHEP 09 (2010) 089, [1006.4945].

[11] C. Bobeth, M. Chrzaszcz, D. van Dyk and J. Virto, Long-distance effects in $B \rightarrow K^{*} \ell \ell$ from analyticity, Eur. Phys. J. C78 (2018) 451, [1707.07305].

[12] N. Gubernari, talk at the workshop "Rare semileptonic B Decays: Theory and Experiment", IPNL Lyon, France, 4-Sep-2019.

[13] LHCB collaboration, R. Aaij et al., Test of lepton universality using $B^{+} \rightarrow K^{+} \ell^{+} \ell^{-}$decays, Phys. Rev. Lett. 113 (2014) 151601, [1406.6482].

[14] LHCB collaboration, R. Aaij et al., Test of lepton universality with $B^{0} \rightarrow K^{* 0} \ell^{+} \ell^{-}$decays, JHEP 08 (2017) 055, [1705.05802].

[15] BABAR collaboration, J. P. Lees et al., Evidence for an excess of $\bar{B} \rightarrow D^{(*)} \tau^{-} \bar{v}_{\tau}$ decays, Phys. Rev. Lett. 109 (2012) 101802, [1205.5442].

[16] BABAR collaboration, J. P. Lees et al., Measurement of an Excess of $\bar{B} \rightarrow D^{(*)} \tau^{-} \bar{v}_{\tau}$ Decays and Implications for Charged Higgs Bosons, Phys. Rev. D88 (2013) 072012, [1303.0571].

[17] BELLE collaboration, M. Huschle et al., Measurement of the branching ratio of $\bar{B} \rightarrow D^{(*)} \tau^{-} \bar{v}_{\tau}$ relative to $\bar{B} \rightarrow D^{(*)} \ell^{-} \bar{v}_{\ell}$ decays with hadronic tagging at Belle, Phys. Rev. D92 (2015) 072014 , [1507.03233].

[18] BELLE collaboration, Y. Sato et al., Measurement of the branching ratio of $\bar{B}^{0} \rightarrow D^{*+} \tau^{-} \bar{v}_{\tau}$ relative to $\bar{B}^{0} \rightarrow D^{*+} \ell^{-} \bar{v}_{\ell}$ decays with a semileptonic tagging method, Phys. Rev. D94 (2016) 072007, [1607.07923].

[19] Belle collaboration, S. Hirose et al., Measurement of the $\tau$ lepton polarization and $R\left(D^{*}\right)$ in the decay $\bar{B} \rightarrow D^{*} \tau^{-} \bar{v}_{\tau}$, Phys. Rev. Lett. 118 (2017) 211801, [1612.00529].

[20] LHCB collaboration, R. Aaij et al., Measurement of the ratio of branching fractions $\mathscr{B}\left(\bar{B}^{0} \rightarrow D^{*+} \tau^{-} \bar{v}_{\tau}\right) / \mathscr{B}\left(\bar{B}^{0} \rightarrow D^{*+} \mu^{-} \bar{v}_{\mu}\right)$, Phys. Rev. Lett. 115 (2015) 111803, [1506.08614].

[21] LHCB collaboration, R. Aaij et al., Measurement of the ratio of the $B^{0} \rightarrow D^{*-} \tau^{+} v_{\tau}$ and $B^{0} \rightarrow D^{*-} \mu^{+} v_{\mu}$ branching fractions using three-prong $\tau$-lepton decays, Phys. Rev. Lett. 120 (2018) 171802, [1708.08856]. 
[22] MILC collaboration, J. A. Bailey et al., $B \rightarrow D \ell v$ form factors at nonzero recoil and $\left|V_{c b}\right|$ from 2+1-flavor lattice QCD, Phys. Rev. D92 (2015) 034506, [1503.07237].

[23] HPQCD collaboration, H. Na, C. M. Bouchard, G. P. Lepage, C. Monahan and J. Shigemitsu, $B \rightarrow D l v$ form factors at nonzero recoil and extraction of $\left|V_{c b}\right|$, Phys. Rev. D92 (2015) 054510 , [1505.03925].

[24] F. U. Bernlochner, Z. Ligeti, M. Papucci and D. J. Robinson, Combined analysis of semileptonic B decays to $D$ and $D^{*}: R\left(D^{(*)}\right),\left|V_{c b}\right|$, and new physics, Phys. Rev. D95 (2017) 115008, [1703.05330].

[25] D. Bigi, P. Gambino and S. Schacht, $R\left(D^{*}\right),\left|V_{c b}\right|$, and the Heavy Quark Symmetry relations between form factors, JHEP 11 (2017) 061, [1707.09509].

[26] J. Aebischer, W. Altmannshofer, D. Guadagnoli, M. Reboud, P. Stangl and D. M. Straub, B-decay discrepancies after Moriond 2019, Eur. Phys. J. C 80 (2020) no.3, 252 [ 1903.10434 ] .

[27] LHCB collaboration, R. Aaij et al., Search for lepton-universality violation in $B^{+} \rightarrow K^{+} \ell^{+} \ell^{-}$decays, Phys. Rev. Lett. 122 (2019) 191801, [1903.09252].

[28] M. Bordone, G. Isidori and A. Pattori, On the Standard Model predictions for $R_{K}$ and $R_{K^{*}}$, Eur. Phys. J. C76 (2016) 440, [1605.07633].

[29] BELLE collaboration, A. Abdesselam et al., Test of lepton flavor universality in $B \rightarrow K^{*} \ell^{+} \ell^{-}$decays at Belle, 1904.02440.

[30] ATLAS collaboration, M. Aaboud et al., Study of the rare decays of $B_{s}^{0}$ and $B^{0}$ mesons into muon pairs using data collected during 2015 and 2016 with the ATLAS detector, Submitted to: JHEP (2018), [1812.03017].

[31] CMS collaboration, S. Chatrchyan et al., Measurement of the B(s) to mu+mu- branching fraction and search for BO to mu+ mu- with the CMS Experiment, Phys. Rev. Lett. 111 (2013) 101804, [1307.5025].

[32] CMS, LHCB collaboration, V. Khachatryan et al., Observation of the rare $B_{s}^{0} \rightarrow \mu^{+} \mu^{-}$decay from the combined analysis of CMS and LHCb data, Nature 522 (2015) 68-72, [1411.4413].

[33] LHCB collaboration, R. Aaij et al., Measurement of the $B_{s}^{0} \rightarrow \mu^{+} \mu^{-}$branching fraction and effective lifetime and search for $B^{0} \rightarrow \mu^{+} \mu^{-}$decays, Phys. Rev. Lett. 118 (2017) 191801, [1703. 05747 ].

[34] C. Bobeth and U. Haisch, New Physics in $\Gamma_{12}^{s}:(\bar{s} b)(\bar{\tau} \tau)$ Operators, Acta Phys. Polon. B44 (2013) 127-176, [1109.1826].

[35] A. Crivellin, C. Greub, D. Müller and F. Saturnino, Importance of Loop Effects in Explaining the Accumulated Evidence for New Physics in B Decays with a Vector Leptoquark, Phys. Rev. Lett. 122 (2019) 011805, [1807.02068].

[36] A. J. Buras, J. Girrbach-Noe, C. Niehoff and D. M. Straub, $B \rightarrow K^{(*)} v \bar{v}$ decays in the Standard Model and beyond, JHEP 02 (2015) 184, [1409.4557].

[37] R. Alonso, B. Grinstein and J. Martin Camalich, Lepton universality violation and lepton flavor conservation in B-meson decays, JHEP 10 (2015) 184, [1505. 05164 ].

[38] L. Calibbi, A. Crivellin and T. Ota, Effective Field Theory Approach to $b \rightarrow s \ell \ell^{(\prime)}, B \rightarrow K^{(*)} v \bar{v}$ and $B \rightarrow D^{(*)} \tau \nu$ with Third Generation Couplings, Phys. Rev. Lett. 115 (2015) 181801, [1506. 02661$]$.

[39] L. Di Luzio, A. Greljo and M. Nardecchia, Gauge leptoquark as the origin of B-physics anomalies, Phys. Rev. D96 (2017) 115011, [1708.08450]. 
[40] N. Assad, B. Fornal and B. Grinstein, Baryon Number and Lepton Universality Violation in Leptoquark and Diquark Models, Phys. Lett. B777 (2018) 324-331, [1708.06350].

[41] L. Calibbi, A. Crivellin and T. Li, Model of vector leptoquarks in view of the B-physics anomalies, Phys. Rev. D98 (2018) 115002, [1709.00692].

[42] M. Bordone, C. Cornella, J. Fuentes-Martin and G. Isidori, A three-site gauge model for flavor hierarchies and flavor anomalies, Phys. Lett. B779 (2018) 317-323, [1712 . 01368].

[43] R. Barbieri and A. Tesi, B-decay anomalies in Pati-Salam SU(4), Eur. Phys. J. C78 (2018) 193, [1712.06844].

[44] A. Greljo and B. A. Stefanek, Third family quark-lepton unification at the TeV scale, Phys. Lett. B782 (2018) 131-138, [1802.04274].

[45] M. Blanke and A. Crivellin, B Meson Anomalies in a Pati-Salam Model within the Randall-Sundrum Background, Phys. Rev. Lett. 121 (2018) 011801, [1801.07256].

[46] B. Fornal, S. A. Gadam and B. Grinstein, Left-Right SU(4) Vector Leptoquark Model for Flavor Anomalies, Phys. Rev. D99 (2019) 055025, [1812.01603].

[47] C. Cornella, J. Fuentes-Martin and G. Isidori, Revisiting the vector leptoquark explanation of the B-physics anomalies, JHEP 07 (2019), 168 [ 1903 . 11517] .

[48] D. A. Faroughy, A. Greljo and J. F. Kamenik, Confronting lepton flavor universality violation in B decays with high-p $p_{T}$ tau lepton searches at LHC, Phys. Lett. B764 (2017) 126-134, [1609. 07138].

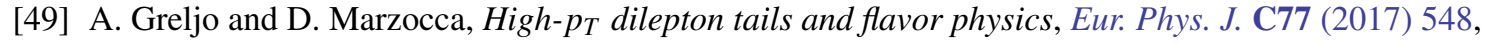
[1704.09015].

[50] B. Diaz, M. Schmaltz and Y.-M. Zhong, The leptoquark Hunter's guide: Pair production, JHEP 10 (2017) 097, [1706.05033].

[51] A. Angelescu, D. Becirevic, D. A. Faroughy and O. Sumensari, Closing the window on single leptoquark solutions to the B-physics anomalies, JHEP 10 (2018) 183, [1808 . 08179 ].

[52] A. Greljo, J. Martin Camalich and J. D. Ruiz-ÃAlvarez, Mono- $\tau$ Signatures at the LHC Constrain Explanations of B-decay Anomalies, Phys. Rev. Lett. 122 (2019) no.13, 131803 [1811.07920] .

[53] M. J. Baker, J. Fuentes-Martin, G. Isidori and M. König, High-pT Signatures in Vector-Leptoquark Models, Eur. Phys. J. C 79 (2019) no.4, 334 [1901.10480] .

[54] F. Feruglio, P. Paradisi and A. Pattori, Revisiting Lepton Flavor Universality in B Decays, Phys. Rev. Lett. 118 (2017) 011801, [1606.00524].

[55] F. Feruglio, P. Paradisi and A. Pattori, On the Importance of Electroweak Corrections for B Anomalies, JHEP 09 (2017) 061, [1705.00929]. 